\title{
The Riddoch phenomenon revealed in non-occipital lobe lesions
}

R. J. ZAPPIA, J. M. ENOGH, R. STAMPER, J. Z. WINKELMAN, AND A. J. GAY

From the Departments of Ophthalmology and Neurology and the Oscar. Johnson Institute, Washington University School of Medicine, St. Louis, U.S.A.

Riddoch (I9I7) described an entity which bears his name, "the Riddoch phenomenon". He observed that while patients with restricted visual fields from occipital wounds could frequently see moving objects, they could not see stationary ones in the affected fields. Riddoch divided his cases into three categories:

(I) Those who perceived movement only on the affected side;

(2) Those who perceived both moving and stationary objects (but to a different degree);

(3) Those with no dissociation between moving and stationary objects.

From his analysis of the visual fields in ten patients with occipital lobe lesions, he concluded that visual functions concerned with the recognition of moving objects and those concerned with the perception of stationary objects are dissociated from each other in the occipital cortex. He considered the absence of this dissociation for a period of several months to be a poor prognostic sign, and postulated that if recovery is occurring after injury the appreciation of movement could be expected to return before the recognition of a comparable stationary object.

Riddoch did not confirm his hypothesis by testing patients with lesions elsewhere in the optic pathway, and while he tested a wide spectrum of lesions due to war injuries, he described no other unique or isolated response characteristics.

This paper reports patients with confirmed lesions in areas other than the occipital lobes who had response characteristics similar to those described by Riddoch.

\section{Case reports}

Patient I, a 55-year-old female, was referred to the Eye Consult Service, Washington University School of Medicine, because of inability to see objects on the right side. This field loss had progressed to a point where she was able to perceive only light and dark on that side.

\section{Examination}

The visual acuity was $20 / 15$ in the right eye and $20 / 25$ in the left. The entire ophthalmological examination gave normal results except for the visual fields. 
Goldmann perimetry (Fig. I) revealed a right homonymous hemianopsia to the $\mathrm{I}_{2 e}, \mathrm{III}_{4}$, and $\mathrm{V}_{4} \mathrm{e}$ test objects in both hemianopic fields. In the parts of the defective field where the patient could see a moving target, she could not see the same target if it was not moving (the Riddoch phenomenon). Because of this, further tests (Sunga and Enoch, 1970) as recently developed by one of us (J.M.E.) were carried out.

These tests not only confirmed that the patient exhibited greater sensitivity to a moving test object than to a stable one, but also demonstrated that the dissociation between moving and non-moving test objects was most noticeable in the corridor between the fovea and the blind spot, and that the kinetic field in the right eye had a tendency to contract on repeated testing.
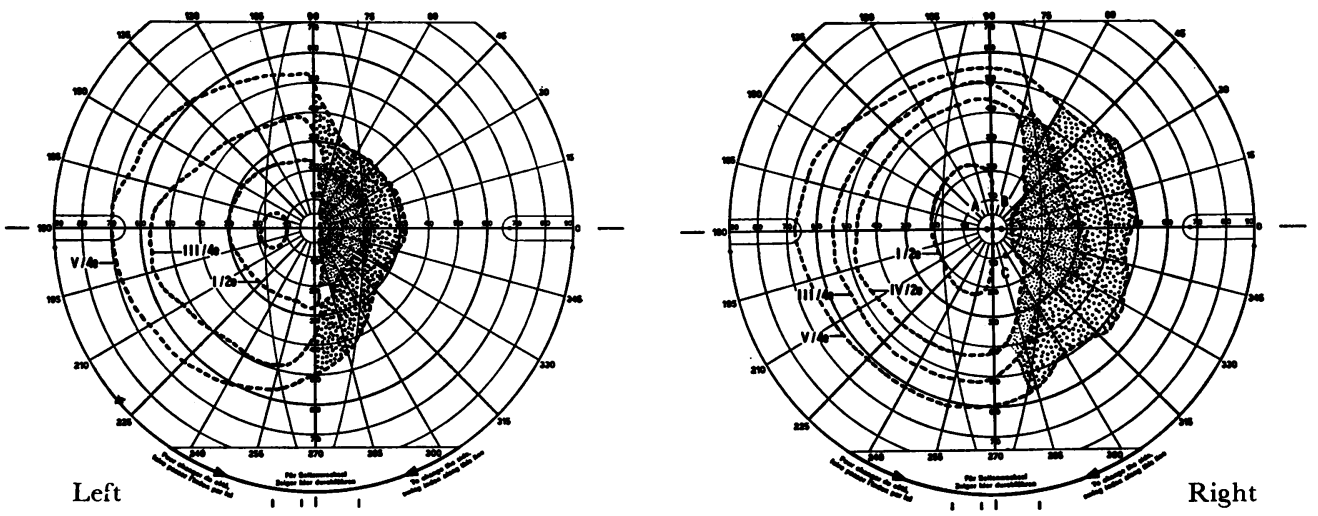

FI G. I Kinetic Goldmann fields illustrating Riddoch phenomenon in Patient $\mathbf{I}$.

Points $A, B$, and $C$ illustrate where repeat flashing static thresholds were determined by 7.M.E. (see Fig. 4)

A repeat flashing static perimetric test was performed using the test technique recently introduced by Sunga and Enoch (1970) (see Addendum). The patient experienced rapid loss of sensitivity to stationary flashing targets as a function of time in affected field areas. Comparable findings have been observed in lesions ranging from optic neuritis to occipital abnormalities (a phenomenon believed to be related, in part, to that described by Riddoch).

On the basis of the right homonymous hemianopsia, the positive Riddoch phenomenon, and the gradual progression by history, a mass lesion in the left occipital lobe was suspected. Neurological examination, skull films, and electroencephalogram were all within normal limits. A lumbar puncture was normal except for a cerebrospinal fluid protein of $60 \mathrm{mg}$./per cent. A left carotid arteriogram revealed a large aneurysm of the internal carotid artery distal to the bifurcation, with an intact posterior cerebral artery coming off the anterior circulation.

\section{Operation}

A large aneurysm of the left internal carotid $(2 \times 3 \mathrm{~cm}$.) was noted to be compressing the left optic tract which was described as "thinned and discoloured". As in the arteriogram, there was no evidence of compression of the posterior cerebral artery.

Patient 2, a 38-year-old male, presented with a complaint of decreased visual acuity in both eyes, with greater loss in the right eye. He had first noticed decreased vision in the right eye in 1965, when he consulted an ophthalmologist who told him that a "blocked blood vessel" was responsible for the visual loss; and 3 months before he was seen in our office, the vision in the left eye had decreased from $20 / 20$ to $20 / 30$.

\section{Examination}

The visual acuity was counting fingers in the right eye and 20/30 in the left. The right pupil exhibited a Gunn phenomenon. Funduscopic examination showed bilateral optic atrophy, much greater on the right side. 
Goldmann perimetry (Fig. 2) revealed a bitemporal hemianopsia with a positive Riddoch phenomenon to a $\mathrm{V}_{4 \mathrm{e}}$ test object in the temporal field of the left eye only.
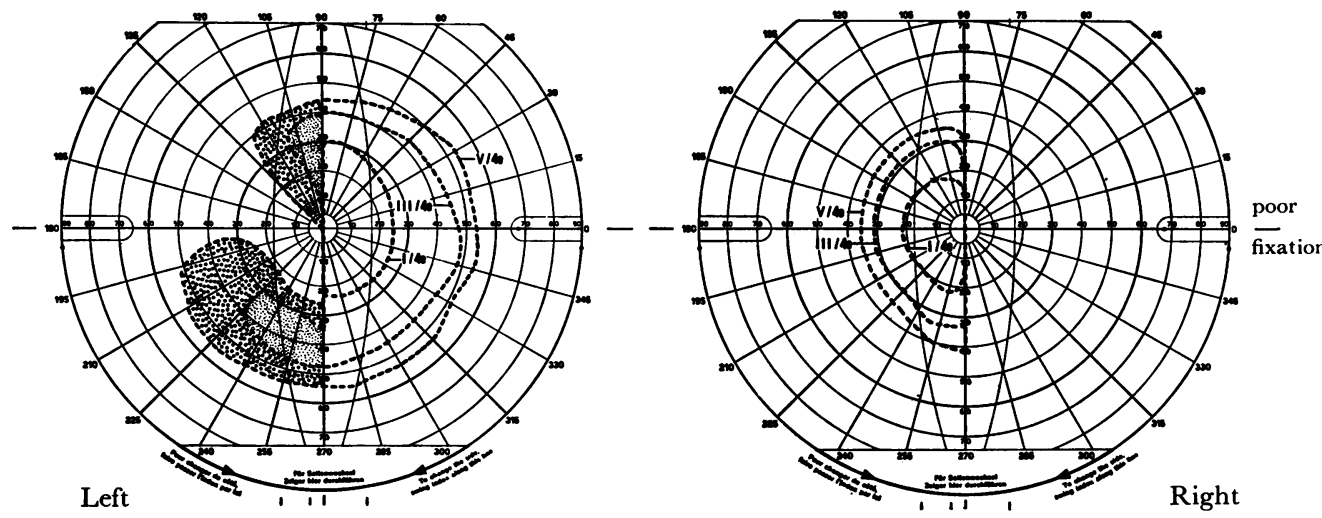

FIG. 2 Preoperative fields, showing field defect in Patient 2, who showed then Riddoch phenomenon only in left eye

Skull $x$ rays showed an enlarged sella turcica. A right carotid arteriogram was felt to be compatible with a tumour in the suprasellar region with anterior extension.

\section{Operation}

The optic chiasm was displaced by a large tumour mass and the right optic nerve was compressed and thinned. Postoperative Goldmann perimetry (Fig. 3) showed a positive Riddoch phenomenon in both temporal fields to the $\mathrm{III}_{4} \mathrm{e}$ and $\mathrm{V}_{4 \mathrm{e}}$ test objects.
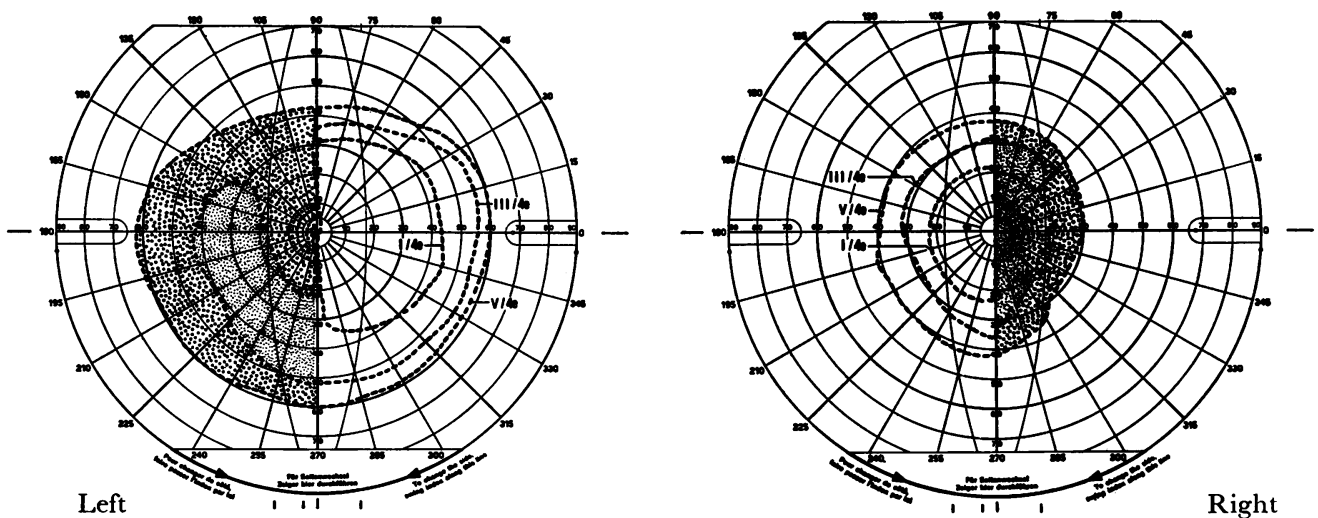

FIG. 3 Postoperative fields of Patient 2, who then displayed bilateral Riddoch phenomenon

\section{Comment}

Walsh and Hoyt (I969) list dissociation of visual perception (Riddoch) under the subheading "Topical Diagnosis of Lesions involving the Optic Radiations and Visual Cortex". Riddoch's hypothesis, however, involved only the visual cortex-not the optic radiations or prestriate area.

Recent extensive neurophysiological evidence (Hubel, 1967) does, indeed, suggest separate loci for different visual functions in the occipital cortex. Riddoch's sign, however, is not pathognomonic for an occipital lobe lesion, nor does it carry the prognostic 
significance that Riddoch attached to it-as evidenced by the two patients we have described and the pathology they demonstrated.

One could hypothesize that, rather than demonstrating a separation of function, Riddoch's data demonstrate that the response to a moving test object is more resistant to loss than the response to a stationary target. There may be some relation between the Riddoch phenomenon and previous studies in local adaptation (Bay, I953; Cibis, 1947, I948) and, more recently, in the work of Enoch and Sunga (1969) and Sunga and Enoch (1970) with repeat static perimetric testing in lesions of the optic pathway.

\section{Summary}

Riddoch (1917) described the dissociation in perception between stationary and moving objects in patients with occipital lobe lesions from war injuries, and commented on the prognosis of patients with field loss who demonstrated this phenomenon. This communication describes two patients with progressive lesions elsewhere in the visual pathway who demonstrated the Riddoch phenomenon, thus showing that the Riddoch phenomenon is not pathognomonic for occipital lobe or optic radiation lesions.

\section{ADDENDUM}

REPEAT STATIC Flashing PERIMETRIC TEST (Fig. 4).

In this test (Enoch and Sunga, 1969; Sunga and Enoch, 1970) the patient is given 5 or more minutes' rest with the eyes closed while sitting in front of a quantitative perimeter. Then, after taking up fixation, he judges the presence or absence of a repeatedly flashing test target located at a predesignated test point in the field. The static threshold is determined again and again for a 5 -minute

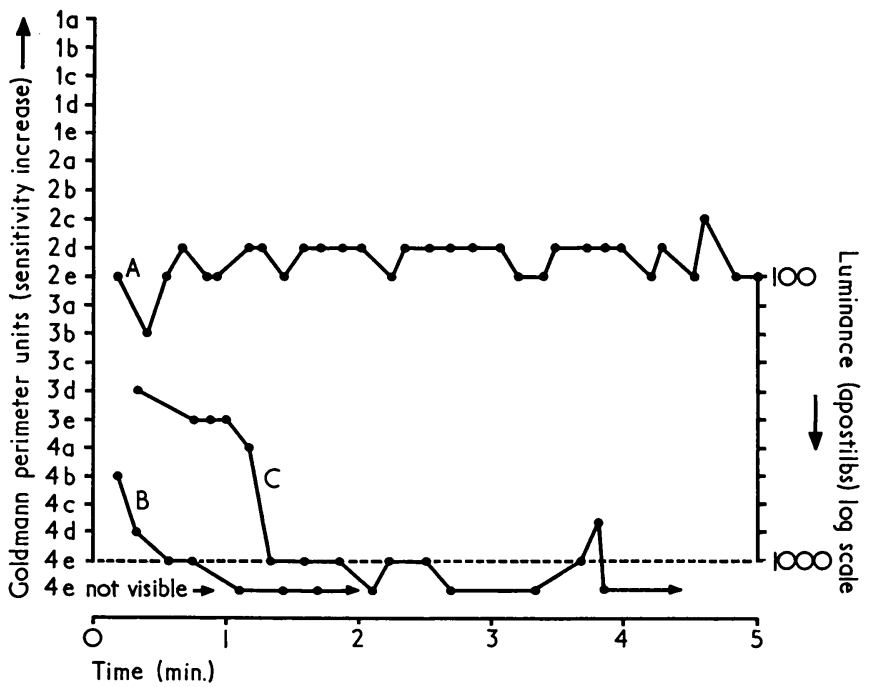

FI G. 4 Repeat flashing static perimetric test utilizing Points $A, B$, and $C$, in the right field of Patient $\mathbf{1} . \quad$ Data have been plotted in terms of visual sensitivity (1/threshold). Perimeter background $3 \mathbf{I} \cdot 5$ apostilbs. Target luminance given on ordinate to right (increasing downwards).

Usual designation of stimulus magnitudes available on Goldmann Haag-Streit perimeter given on ordinate to left. Here the threshold to a periodically presented non-moving stimulus has been determined. The same test is repeated again and again during a 5-min. test period (Sunga and Enoch, 1970; Enoch and Sunga, 1969).

${ }_{4} \mathrm{~N} V$ signifies that even an I 000 apostilb target ( I oo millilamberts) was "not visible" to the patient. 
period at this point. The light is flashed in order to distinguish a saturation or fatigue-like effect from a local adaptation anomaly.

The first patient referred to above was tested initially at Point $A$ (Fig. I) in the normal part of the field, using a Goldmann size o target presented for $70 \mathrm{msec}$. every half second. In Fig. 4, we see that Curve $A$ gives a relatively stable, normal response throughout the 5-minute test session. Repeating the test at Point $B$ (Fig. I), a Goldmann size $\mathrm{V}$ target was required! A marked decay in sensitivity was noted at this point (Fig. 4). The test was repeated at Point $C$ (Fig. I), using a Goldmann size IV target. At this locus, the initial sensitivity was higher, but it again fell off sharply in time (Fig. 4). $4 e \mathcal{N V}$ in Fig. 4 signifies that the brightest target in the Goldmann perimeter ( 1 ,ooo apostilbs) was no longer visible.

\section{References}

BAY, E. (1953) Brain, 76, $5^{1} 5$

CIBIS, P. (1947) v. Graefes Arch. Ophthal., 148, I (1948) Ibid., 148, 2 1 6

eNOCH, J. M., and SUNGA, R. N. (I969) Docum. ophthal. (Den Haag), 26, 2 I 5

HUBEL, D. H. (1967) Physiologist, 10, 17

RIDDOGH, G. (1917) Brain, 40, I5

SUnGa, R. n., and ENOCH, J. м. (1970) Amer. F. Ophthal., 70, 403

WAlsh, F. в., and ноYт, w. F. (1969) "Clinical Neuro-ophthalmology", 3rd ed., vol. I, p. 77.

Williams and Wilkins, Baltimore. 